\title{
A comparative study of highlights detection and elimination by color morphology and polar color models
}

\author{
Francisco Ortiz, Fernando Torres, and Pablo Gil \\ Automatics, Robotics and Computer Vision Group. \\ Dept. Physics, Systems Engineering and Signal Theory. University of Alicante, \\ P.O. Box 99, 03080 Alicante, Spain. \\ \{fortiz, Fernando.torres, Pablo.Gil\}@ua.es
}

\begin{abstract}
In this paper, we present a comparative study of detection and elimination of highlights in real color images of any type of material. We use different polar color spaces for the automatic highlight detection (HLS, HSV and L1-norme). To eliminate the highlights detected, we use a new connected vectorial filter of color mathematical morphology which it operates exclusively on bright zones, reducing the high cost of processing of the connected filter and avoiding over-simplification. The new method proposed here achieves good results and it not requires costly multiple-view systems or stereo images.
\end{abstract}

\section{Introduction}

In visual systems, images are acquired in work environments in which illumination plays an important role. Sometimes a bad adjustment of the illumination can introduce highlights (brightness or specular reflectance) into the objects captured by the vision system. Highlights in images have long been disruptive to computer-vision algorithms. They appear as surface features, which can lead to problems, such as stereo mismatching, false segmentation and recognition errors. In spite of such undesirable effects, however, there is, so far, no application available in commercial software that allows the automatic detection and elimination of such specularities.

To effectively eliminate the highlights in captured scenes, we must first identify them. The dichromatic reflection model, proposed by Safer [1], is one tool that has been used in many methods for detecting specularities. It supposes that the interaction between the light and any dielectric material produces different spectral distributions within the object (specular and diffuse reflectance). The specular reflectance has the same spectral makeup as the incident light, whereas, the diffused component is a product of illumination and surface pigments. Based on this model, Lin et al [2] have developed a system for eliminating specularities in image sequences by means of stereo correspondence. Bajcsy et al [3] use a chromatic space based on polar coordinates that allows the detection of specular and diffuse reflections by means of the previous knowledge of the captured scene. Klinker et al [4] employ a pixelclustering algorithm that has been shown to work well in detecting brightness in images of plastic objects. 
These above-mentioned approaches have produced good results but they entail requirements that limit their applicability, such as the use of stereo or multiple-view systems, long processing time, the previous knowledge of the scene, etc. Furthermore, some techniques merely detect brightness without eliminating it.

The organization of this paper is as follows: In Section 2, we present the color models for processing, together with the bi-dimensional diagrams used to detect the specular reflectance (highlights). In Section 3, we show the extension of the geodesic operations to color images. Section 4 we present the detection of highlights and our experimental results. The elimination process is presented in Section 5. Finally, our conclusions are outlined in the last section.

\section{Polar representations for highlight detection}

In the last years, the color spaces based in polar coordinates are widely used in image processing. Important advantages of these color spaces are: good compatibily with human intuition of colors and separability of chromatic values from achromatic values. The two classic polar spaces are HLS and HSV [5,6]. The HLS and HSV components are calculated from RGB system. The formulas change from cartesian co-ordinates to polar co-ordinates. The luminance $l$ and saturation $s$ of HLS are calculated as follows:

$$
\left\{\begin{array}{l}
l=\frac{\max (r, g, b)+\min (r, g, b)}{2} \\
s= \begin{cases}\frac{\max (r, g, b)-\min (r, g, b)}{\max (r, g, b)+\min (r, g, b)} & \text { if } l \leq 0.5 \\
\frac{\max (r, g, b)-\min (r, g, b)}{2-\max (r, g, b)+\min (r, g, b)} & \text { if } l>0.5\end{cases}
\end{array}\right.
$$

where $r, g, b$ of RGB, and $s$ and $l$ range from 0 to 1 . The HLS representation has cylindrical shape with the previous formulas. The HSV has a cone shape and the coordinates of value $v$ and saturation $s$ are calculated as follows:

$$
\left\{\begin{array}{l}
v=\max (r, g, b) \\
s=\frac{\max (r, g, b)-\min (r, g, b)}{\max (r, g, b)}
\end{array}\right.
$$

We propose to exploit the existing relation of the specularities presents in a color image with specific coordinates of $l$ and $s$ in HLS or $v$ and $s$ in HSV, independently of the hue of the object in which the highlights appears [7]. These representations of colors are bi-dimensional histograms with a grey value, in which each co-ordinate $(l, s)$ or $(v, \mathrm{~s})$ indicates the amount of pixels with $l$ and $s$ (HLS) or $v$ and $s$ (HSV) in the original color image. Figure 1.a and Figure 1.b show the LS diagram and VS diagram for HLS and HSV color spaces, respectively. 
The two polar systems (HLS and HSV) have some in-coherences that prevent the use of these color representations in some image processing. This is due to the conversion formulas from RGB to polar spaces. Some instability arises in saturation of HLS and HSV spaces for small variations of RGB values. In addition, the saturation of the primary colors is not visually agreeable in HLS and HSV. In order to avoid these inconveniences we also use the Serra's L1-norme [8], where the intensity (achromatic) signal $m$ and saturation signal $s$ are calculated as follows:

$$
\begin{cases}m= & \frac{1}{3}(r+g+b) \\ s= \begin{cases}\frac{1}{2}(2 r-g-b)=\frac{3}{2}(r-m) & \text { if }(b+r) \geq 2 g \\ \frac{1}{2}(r+g-2 b)=\frac{3}{2}(m-b) & \text { if }(b+r)<2 g\end{cases} \end{cases}
$$

Figure 1.c shows the MS diagram from Serra's L1-norme as a positive projection of all the corners of the RGB cube in a normalization of the achromatic line to the $m$ signal.

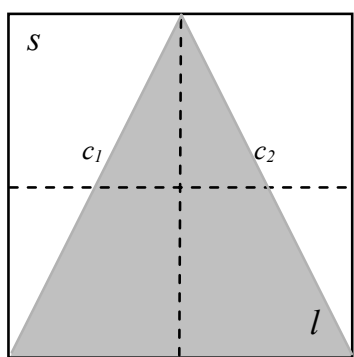

(a)

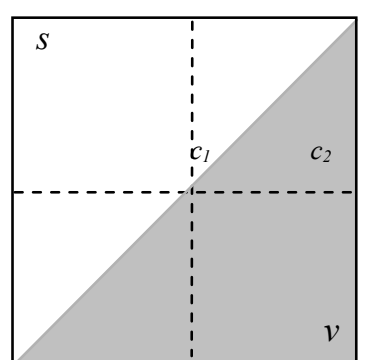

(b)

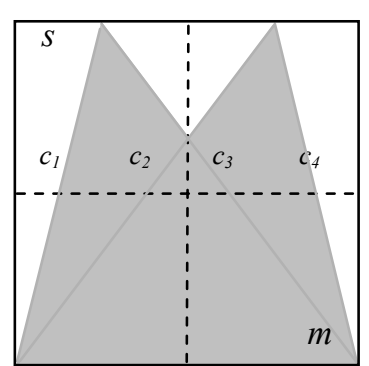

(c)

Fig.1. 2D bi-dimensional diagrams of HLS (a), HSV (b) and L1-norme (c). Positive projection.

\section{Vector connected filters}

Morphological filters by reconstruction have the property of suppressing details while preserving the contours of the remaining objects $[9,10]$. The use of such filters in color images requires an ordered relationship among the pixels of the image. For the vectorial morphological processing, the lexicographical ordering $o_{\mathrm{lex}}=$ achromatic value $\rightarrow$ saturation $\rightarrow$ hue, will be used $[11,12]$.

Once the orders have been defined, the morphological operators for the reconstruction of color images can be applied. Geodesic dilation is an elementary geodesic operation. Let $\boldsymbol{g}$ denote a marker color image and $\boldsymbol{f}$ a mask color image (if $o_{\text {lex }}(\boldsymbol{g}) \leq o_{\text {lex }}(\boldsymbol{f})$, then $\left.\boldsymbol{g} \wedge_{v} \boldsymbol{f}=\boldsymbol{g}\right)$. The vectorial geodesic dilation of size 1 of the marker image $\boldsymbol{g}$ with respect to the mask $\boldsymbol{f}$ can therefore be defined as: 


$$
\delta_{v}{ }_{f}^{(1)}(g)=\delta_{v}^{(1)}(g) \wedge v f
$$

where $\delta_{v}{ }^{(1)}(g)$ is the vectorial dilation of size 1 of the marker image $g$.

The vectorial geodesic dilation of size $n$ of a marker color image $g$ with respect to a mask color image $\boldsymbol{f}$ is obtained by performing $n$ successive geodesic dilations of $\boldsymbol{g}$ with respect to $f$ :

$$
\delta_{v}{ }_{\boldsymbol{f}}^{(n)}(\boldsymbol{g})=\delta_{v}{ }_{\boldsymbol{f}}^{(1)}\left[\delta_{v \boldsymbol{f}}^{(n-1)}(\boldsymbol{g})\right]
$$

with $\delta_{v}{ }_{\boldsymbol{f}}^{(0)}(\boldsymbol{g})=\boldsymbol{f}$.

Geodesic transformations of images always converge after a finite number of iterations. The propagation of the marker image is limited by the mask image. Morphological reconstruction of a mask image is based on this principle [13].

The vectorial reconstruction by dilation of a mask color image $\boldsymbol{f}$ from a marker color image $\boldsymbol{g}$, (both with $D_{f}=D_{g}$ and $\left.o_{l e x}(\boldsymbol{g}) \leq o_{l e x}(\boldsymbol{f})\right)$ can be defined as:

$$
R_{v} \boldsymbol{f}(\boldsymbol{g})=\delta_{v_{\boldsymbol{f}}}^{(n)}(\boldsymbol{g})
$$

where $n$ is such that $\delta_{v}{ }_{\boldsymbol{f}}^{(n)}(\boldsymbol{g})=\delta_{v}{ }_{\boldsymbol{f}}^{(n+1)}(\boldsymbol{g})$.

\section{Highlight detection by HLS, HSV and L1-norme}

Androutsos et al in [14] make a division of a luminance-saturation space and they conclude that if the saturation is greater than a $20 \%$ and the luminance is greater than a $75 \%$, the pixels are chromatic, if the saturation is smaller than a $20 \%$ and the luminance is greater than $75 \%$, the pixels are luminous or highlights. Our criterion is similar and it is based on the division of the LS, VS and MS diagrams in different homogenous regions that segment the pixels of the chromatic image. The exact limits of the regions must be calculated empirically, and in this comparative study we will show the exact values for the brightness region in the HLS, HSV and the L1-norme.

Not all the images have the same dynamic range and, therefore, the specularities do not present the same achromatic values and saturations. The best solution for this problem is to apply a vector morphological contrast enhancement for luminous pixels. We denote the color morphological contrast enhancement by:

$$
\boldsymbol{f}^{\prime}=\boldsymbol{f}+W_{T H}(\boldsymbol{f})
$$

where $\boldsymbol{f}$ 'is the new contrasted color image and $W T H_{v}(f)$ is the vectorial top-hat $(\boldsymbol{f}$ $\left.\gamma_{v}(f)\right)$ of the original color image $f$. The color morphological contrast enhancement expels only the highlights to the limits of the RGB cube. The result of the local enhancement by the top-hat is that the specular reflectance pixels are located on $c_{2}$ 
line in LS and VS diagrams. In MS diagram the bright pixels are located on the $c_{3}$ and $c_{4}$ lines. The $s_{\max }$ value will be shown in the next section.

\subsection{Use of LS, VS and MS diagrams for highlight detection}

We present the results of a study for highlight detection carried out on a set of real chromatic images that are quite representative of countless common materials (i.e., plastic, ceramics, fruit, wood, etc.), in which there are strong and weak reflectances. A subset of the images used in the study and its bi-dimensional diagrams (LS, VS, and MS) are present in Figure 2.

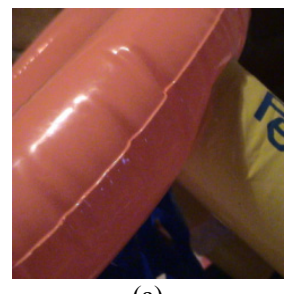

(a)

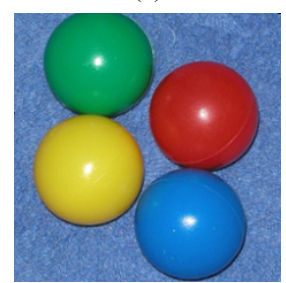

(e)

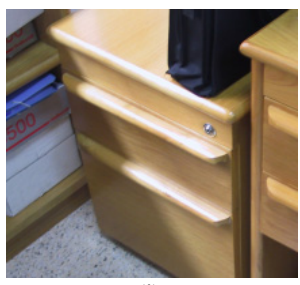

(i)

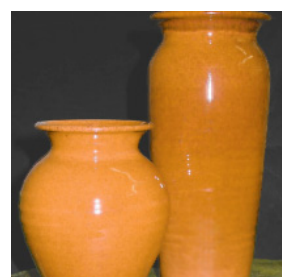

(m)

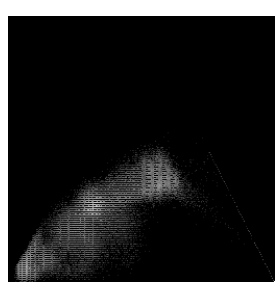

(b)

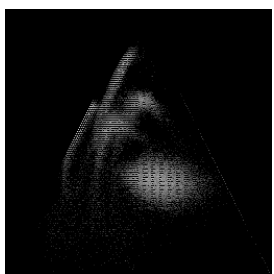

(f)

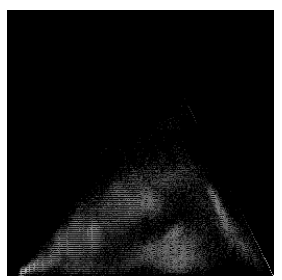

(j)

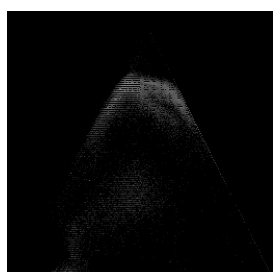

(n)

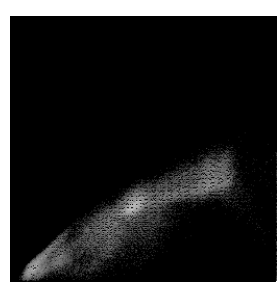

(c)

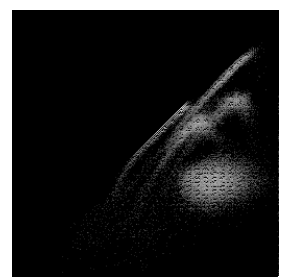

(g)

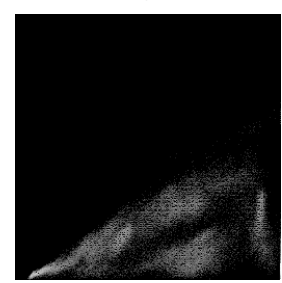

(k)

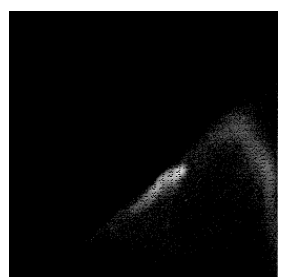

(o)

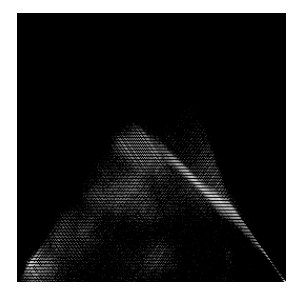

(d)

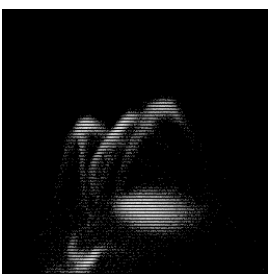

(h)

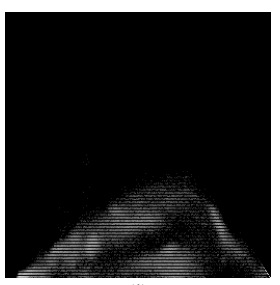

(1)

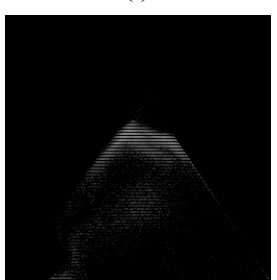

(p)

Fig.2. Color images for empirical study and bi-dimensional histograms. "Life-saver" (a), "Balloons" (e), "Drawers" (i) and "Vases" (m). LS diagrams in (b), (f), (j) and (n). VS diagrams in (c), (g), (k) and (o). MS diagrams in (d), (h), (l) and (p). 
Figure 3 shows the evolution of the highlights detected in Fig. 2 when the saturation $s$ is increased along $c_{2}$ line (HLS and HSV), or $c_{3}$ and $c_{4}$ lines (MSH). It is a logarithmic evolution where most of the bright pixels are located in maximum value of achromatic signal and minimum $s$ (up to $80 \%$ ). The rest correspond to the transition from specular to diffuse reflection of the dichromatic refection model [1] in the surface of the objects. The graphs show that the detection of specularities stops, in all of the cases, at a maximum saturation of $s_{\max }=25(10 \%$ of 255$)$. In HSV, $s_{\max }$ is smaller or equal to 18 .

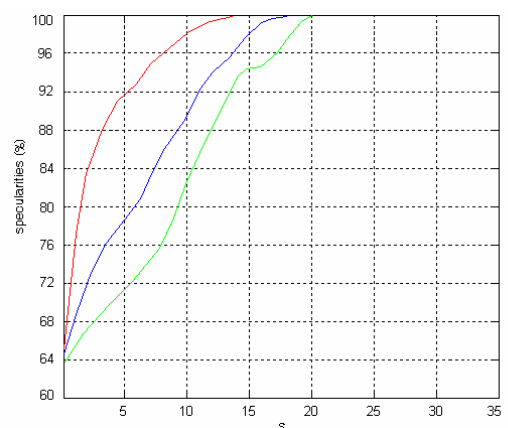

(a)

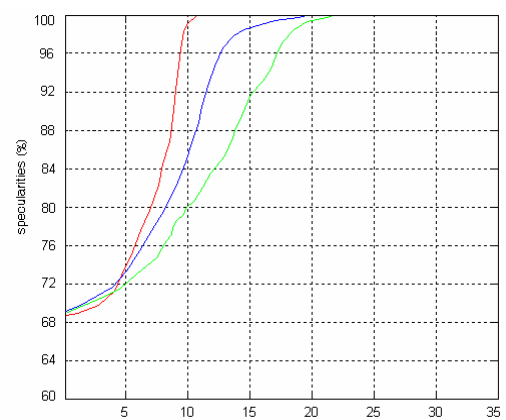

(c)

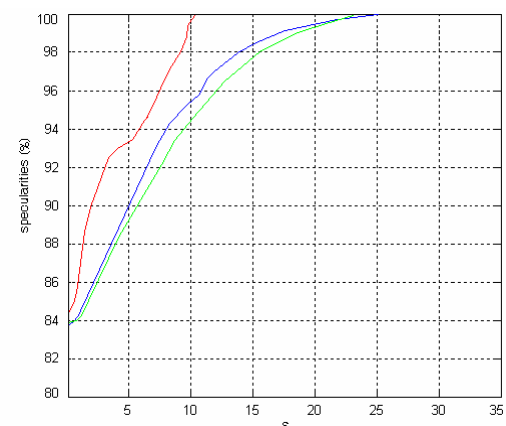

(b)

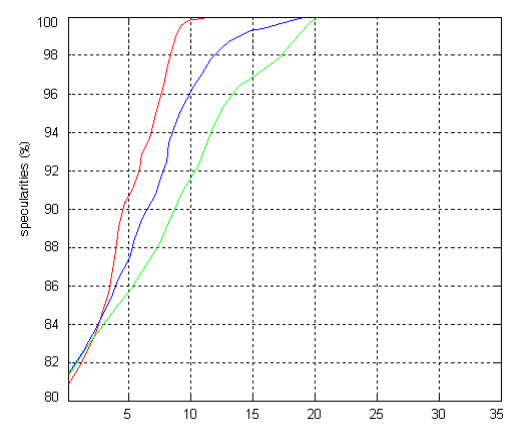

(d)

Fig.3. Evolution (\%) of highlights detected in bi-dimensional diagrams by saturation value $s$, in HSV (red line), HLS (blue line) and MSH (green line). (a) "Life-saver", (b) "Balloons", (c) "Drawers" and (d) "Vases".

\section{Highlight elimination by vector connected filters}

To eliminate the highlight that which was previously detected with the previous diagrams, we propose the use of geodesic filters of mathematical morphology [15]. Specifically, a vectorial opening by reconstruction applied exclusively to the specular areas of the image and their surroundings. In the filter, we use vector ordering $o_{\text {lex }}=m \rightarrow s \rightarrow h$ (L1-norme). A new mask-image $h(x, y)$ represents the pixels of $\boldsymbol{f}$ with which we will operate. The mask-image $h$ is a dilation of the mask of highlights 
detected. Assuming $D_{h}=D_{f}$, each pixel $(x, y)$ has a value of $h(x, y)=\{0,1\}$, where $h(x, y)=1$ in the new areas of interest in the image. The size $n$ of the structural element of the dilation will determine the success of the reconstruction and the final cost of the operations, since this size defines the area to be processed by the filters. In the geodesic filter, $\boldsymbol{f}$ is first eroded with a structuring element of size $e$. The new filter is defined, considering that, in this case, the operation will not affect all the pixels $(x, y)$, but only those in which $h(x, y)=1$ :

$$
R_{v} \boldsymbol{f}, h=\left\{\delta_{v \boldsymbol{f}}^{(n)}\left(\varepsilon_{v}^{(e)}(\boldsymbol{f})\right) \mid \forall \boldsymbol{f}(x, y) \Rightarrow h(x, y)=1\right\}
$$

where $n$ is such that $\delta_{v \mathbf{f}}{ }^{(n)}\left(\varepsilon_{v}{ }^{(e)}(\boldsymbol{f})\right)=\delta_{v \mathbf{f}}{ }^{(n+1)}\left(\varepsilon_{v}{ }^{(e)}(\boldsymbol{f})\right)$. If there is not brightness in the original image then, the reconstruction is not made.

From the results in Figure 4, the effectiveness of our method for the detection and elimination of specular reflectance can be observed. The over-simplification does not appear with the new filter, since the reconstruction only functions in bright areas. Furthermore, the results are obtained at a much lower computational time.

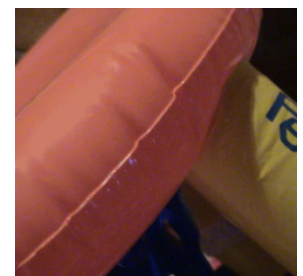

(a)

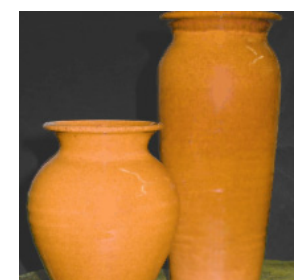

(c)

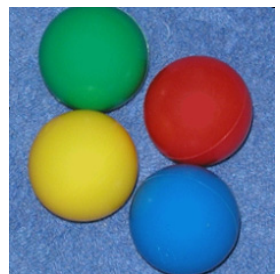

(b)

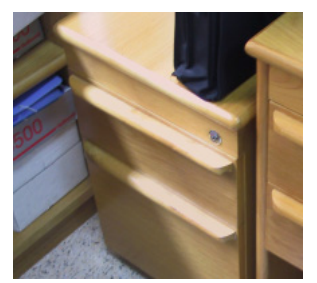

(d)

Fig. 4. Highlight elimination of real color images of Figure 2. Over-simplification is not present in the results.

\section{Conclusions}

In this paper, we have presented a comparative study about the detection and elimination of highlights in color images by color spaces based on polar co-ordinates. A detailed analysis has demonstrated that the brightness appear in a given area of the 
LS, VS and MS diagrams. The three spaces can be used, although the MSH allows the detection of brightness by different hue.

The use of a new connected vectorial filter allows us to eliminate the specular reflectance (highlights) previously detected. This filter is an extension of the geodesic transformations of the mathematical morphology to color images. The possibility of eliminating brightness in color images without causing over-simplification, has also been demonstrated. In addition, the elimination of brightness has been obtained automatically with a very short processing time. It is a reduction of temporal cost between $50 \%$ and $80 \%$, with respect to a global geodesic reconstruction.

Based on the success shown by these results, the objective is to reduce the processing time required for geodesic operations as much as possible.

\section{References}

1. Shafer, S.A.: Using color to separate reflection components. Color Research Appl. Vol. 10 (1985) 210-218

2. Lin, S., Li, Y., Kang, S., et al: Diffuse-Specular Separation and Depth Recovery from Image Sequences. Lecture Notes in Computer Science, Springer-Verlag. Vol. 2352 (2002).

3. Bajcsy, R., Lee, S., Leonardis, A.: Detection of diffuse and specular interface reflections and inter-reflections by color image segmentation. International Journal on Computer Vision. Vol. 17 (1996) 241-271.

4. Klinker, G., Shafer, S.A., kanade, T.: Image segmentation and reflection analysis through color. In: Proc. SPIE. Vol. 937 (1988) 229-244.

5. Palus, H., Representations of colour images in different colour spaces. In: Sangwine, S., and Horne, R. (eds.): The Colour Image Processing Handbook, Chapman and Hall (1998) 67-90.

6. Plataniotis, K.N., Venetsanopoulos, A.N.: Color Image Processing and Applications, Springer-Verlag Berlin (2000)

7. Torres, F., Angulo, J., Ortiz, F.: Automatic detection of specular reflectance in colour images using the MS diagram. Lecture Notes in Computer Science, Springer-Verlag. Vol. $2756(2003) 132-139$.

8. Serra, J.: Espaces couleur et traitement d'images. Tech. Report N-34/02/MM. Centre de Morphologie Mathématique, École des Mines de Paris (2002)

9. Vicent, L.: Morphological Grayscale Reconstruction in Image Analysis: Applications and Efficient Algoritms. IEEE Transactions on Image Processing. Vol. 2. (1993) 176-201

10. Crespo, J. Serra, J., Schafer, R.: Theoretical aspects of morphological filters by reconstruction. Signal Processing. Vol. 47 (1995) 201-225

11. Ortiz, F., Torres, F., De Juan, E., Cuenca, N.: Colour mathematical morphology for neural image analysis. Journal of Real-Time Imaging. Vol. 8 (2002) 455-465

12. Ortiz F., Torres F., Gil P.: Gaussian noise elimination in colour images by vector-connected filters. In: Proc. IEEE 17th International Conference on Pattern Recognition. Vol. 4 (2004) 807-811

13. Soille, P.: Morphological Image Analysis. Principles and Applications. Springer-Verlag (1999)

14. Androutsos, D., Plataniotis, K., Venetsanopoulos, A.: A novel vector-based approach to color image retrieval using a vector angular-based distance measure. Computer Vision and Image Understanding, Vol. 75 (1999)

15. Ortiz F., Torres, F.: Vectorial morphological reconstruction for brightness elimination in colour images. Journal of Real-Time Imaging. Vol. 8 (2004) 379-387. 